\title{
BMJ Open Cardio-ankle vascular index (CAVI) measured by a new device: protocol for a validation study
}

\author{
Sergio Rico Martín (D) , ,2 Valentina Vassilenko, ${ }^{2,3}$ Jorge M de Nicolás Jiménez, ${ }^{2,4}$ \\ Purificación Rey Sánchez, ${ }_{1}^{1}$ Andreia Serrano, ${ }^{2,3}$ Mariana Martínez Alvarez, ${ }^{5}$ \\ Julián F Calderón García, 1,2 Juan F Sánchez Muñoz-Torrero ${ }^{2,6}$
}

To cite: Rico Martín S, Vassilenko V, de Nicolás Jiménez JM, et al. Cardio-ankle vascular index (CAVI) measured by a new device: protocol for a validation study. BMJ Open 2020;10:e038581. doi:10.1136/ bmjopen-2020-038581

- Prepublication history for this paper is available online. To view these files, please visit the journal online (http://dx.doi. org/10.1136/bmjopen-2020038581).

JFCG and JFSM-T contributed equally.

Received 16 March 2020 Revised 14 September 2020 Accepted 22 September 2020

Check for updates

(C) Author(s) (or their employer(s)) 2020. Re-use permitted under CC BY-NC. No commercial re-use. See rights and permissions. Published by BMJ.

For numbered affiliations see end of article.

Correspondence to Dr Sergio Rico Martín; sergiorico@unex.es

\section{ABSTRACT}

Introduction Cardio-ankle vascular index (CAVI) is a new marker of arterial stiffness (AS) that can assess vascular wall stiffness in the aorta, femoral artery and tibial artery. CAVI is less affected by blood pressure at the time of measurement than the gold standard method (carotid-femoral pulse wave velocity (PWV)). Our group has developed a device called VOPITB (Velocidad Onda de Pulso Índice Tobillo Brazo) that uses the oscillometric method and easily and accurately measures the PWV in the arms and legs separately, allowing new AS indices to be studied. This article describes the research protocol to determine CAVI using VOPITB and to validate the device against a reference device (VaSera VS-1500) and assess its clinical utility.

Methods and analyses A cross-sectional, descriptive and observational study will be conducted. In all, 120 subjects (a minimum of $40 \%$ of subjects from any one gender) will be evaluated. CAVI will be determined from the measurement by VOPITB and VaSera VS-1500. For each subject, the average of the three readings taken with each device will be calculated. The Bland-Altman plot will be used to determine whether any bias exists in the datathat is, a tendency of the size of the difference to vary with the mean. The participants will be divided roughly equally between the following age bands: $<30,30-60$ and $>60$ years.

Ethics and dissemination The study has been approved by the ethics committee of the Hospital San Pedro de Alcántara, Cáceres, Spain. The participants will be required to sign an informed consent form before inclusion in the study, in accordance with the Declaration of Helsinki and WHO standards for observational studies. The dissemination plan of the research study results will be through presentations in relevant national and international conferences and scientific publications in peer-reviewed journals.

Trial registration number NCT04303546.

\section{INTRODUCTION}

Arterial stiffness (AS) is usually related to the structural and functional properties of large arteries, and it is considered a predictor of cardiovascular events. ${ }^{1}{ }^{2}$ Furthermore, AS is likely to predate hypertension and target organ damage, with important implications

\section{Strengths and limitations of this study}

- VOPITB (Velocidad Onda de Pulso Índice Tobillo Brazo) is a new oscillometric device that measures the pulse wave velocity (PWV) in the arms and legs separately.

- Cardio-ankle vascular index (CAVI) is an alternative method to assess arterial stiffness and overcomes some disadvantages of the gold standard method, that is, carotid-femoral PWV.

- This study will validate CAVI using VOPITB against a reference device (VaSera VS-1500) and assess its clinical use.

- Incorporating CAVI into VOPITB functions would complement the diversity of variables that allow the estimation of arterial stiffness.

- Limitations include that the inclusion of muscular arteries may contribute little to the prognostic value of CAVI.

for the early identification of individuals at high risk of cardiovascular disease (CVD). ${ }^{3}$ The elastic wall properties of large arteries are measured by carotid-femoral pulse wave velocity (cfPWV), which is considered the gold standard for AS assessment. ${ }^{145}$ However, the high cost of the commercial equipment available on the market (SphygmoCor, Complior), the discomfort for some patients when exposing the inguinal area and the time needed to perform the examination limit its routine use primarily to research applications. Moreover, the difficulty in locating the carotid and femoral pulses during measurements makes the equipment highly operatordependent. $^{6-8}$ To simplify this technique and consider that any part of the body could be appropriate to assess AS, new methods have been proposed in Japan by measuring PWV in peripheral arteries: cardio-ankle vascular index $(\mathrm{CAVI})^{9}$ and brachial-ankle pulse wave velocity (baPWV). ${ }^{10}$

CAVI is a new marker of $\mathrm{AS}^{9}$ based on the stiffness parameter $\beta^{11}$ and Bramwell-Hill 
formula. ${ }^{12}$ CAVI can quantitatively assess vascular wall stiffness in the aorta, femoral artery and tibial artery by measuring the increase in AS observed from end-diastole to end-systole. ${ }^{9}$ CAVI is less affected by blood pressure (BP) at the time of measurement compared with cfPWV. ${ }^{13} 14$ The measurement method is also simplified using BP cuffs located in both arms and ankles and a microphone on the chest. In addition, it is mainly operator-independent ${ }^{14}$ with good reproducibility. ${ }^{15} \mathrm{~A}$ recent meta-analysis found an association between CAVI and incident CVD. ${ }^{16}$

Our group has developed a device called VOPITB (Velocidad Onda de Pulso Índice Tobillo Brazo), ${ }^{17}$ which uses the oscillometric method to easily and accurately measure PWV in the arms and legs separately, allowing new AS indices to be studied (sum, difference, ratio, baPWV and CAVI). For example, the difference in PWV in the leg and the arm is correlated with the quantification of coronary calcium, ${ }^{18}$ and the sum of PWVs from all the limbs is associated with cardiovascular risk. ${ }^{17}$ Moreover, the clinical use of baPWV measured by VOPITB is similar to that measured by VaSera VS-1500. ${ }^{19}$ Because VOPITB can independently measure PWV in each limb, it is possible to calculate CAVI. Incorporating CAVI into VOPITB functions would complement the diversity of variables that allow the estimation of AS. This article describes the research protocol to determine CAVI using VOPITB and to validate the device against a reference device (VaSera VS-1500) and assess its clinical use.

\section{METHODS AND ANALYSIS}

The experimental protocol was developed according to the recommendations of the ARTERY Society guidelines for the validation of non-invasive haemodynamic measurement devices. ${ }^{20}$

\section{Study setting and design}

This cross-sectional, descriptive and observational study will be conducted at the Hospital Universitario San Pedro de Alcántara of Cáceres and Centro de Salud Zona Centro of Cáceres (Spain). The time estimated for this project is 8 months.

\section{Sample size and study population}

The sample size has been calculated so that a difference between CAVI values obtained from the measurement of both devices, VOPITB and VaSera VS-1500, will be up to 0.8 points compared with the CAVI reference values for the Spanish adult population. ${ }^{21}$ The base parameters used to calculate the sample size were as follows: SD of CAVI measured by VaSera VS-1500 and VOPITB: 1.44; mean difference between CAVI measurements: 0.8; CI: 95\%; and power: $80 \%$. Thus, the estimated sample size was calculated as 102 patients. In addition, to compensate for possible incomplete data sets from patients who could withdraw or fail to complete the study, $20 \%$ were added. Finally, a total of 120 subjects (a minimum of $40 \%$ of subjects from each gender) will be evaluated according to the following criteria:

- <30 years (40 subjects).

- 30-60 years (40 subjects).

- 60 years (40 subjects).

Subjects $<30$ years of age will be recruited from among students of Extremadura University. Subjects 30-60 years of age will be selected from among hospital and university workers. Finally, subjects $>60$ years of age will be recruited from among primary care patients at the health centre. The participants will be required to sign an informed consent form before inclusion in the study, in accordance with the Declaration of Helsinki and WHO standards for observational studies. ${ }^{22}$

\section{Inclusion criteria}

Subjects $\geq 18$ years of age who agree to participate in the study and do not meet any of the exclusion criteria.

\section{Exclusion criteria}

- History of CVD (ischaemic heart disease, stroke or peripheral artery disease (PAD)).

- Atrial fibrillation or other cardiac rhythm disorders.

- Pacemaker dependent.

- Impalpable arterial pulse at the site of measurement.

- PAD: either intermittent claudication with an anklebrachial index (ABI) less than 0.9 or high, or previous vascular intervention or limb amputation for PAD.

- Aortic valve diseases and aortic diseases.

- Subjects with haemodialysis.

- Pregnancy.

- Terminal condition.

- Age $<18$ years.

\section{Patient and public involvement}

Patients and/or the public were not involved in the design, conduction, reporting or dissemination plans of this research.

\section{Variables and measurement instruments}

Information containing general and variable data, such as age, gender, occupation, family history of CVD, cardiovascular risk factors (diabetes, hypertension, dyslipidaemia, obesity, chronic renal failure and tobacco consumption), alcohol consumption and medications and drugs used, will be recorded. We will also collect the start date and doses of medications, namely, antihypertensive, antidiabetic and antiplatelet agents; anticoagulants; and lipidlowering drugs.

Parameters such as weight, height, body mass index (BMI), waist circumference, heart frequency and BP will be determined during physical examination. Height will be measured using a Harpenden stadiometer, and weight will be measured using a biomedical precision balance (Seca 770; Medical Scale and Measurement Systems, Birmingham, UK). Both measurements will be determined only when the participants will be wearing light clothing and no shoes. The BMI will be calculated as the weight in kilograms divided by the square of the height 
Table 1 Order of measurement for two sequential subjects

\begin{tabular}{ll}
\hline Subject 1 & Subject 2 \\
\hline VOPITB, observer A & VaSera VS-1500, observer A \\
\hline VaSera VS-1500, observer B & VOPITB, observer B \\
\hline VOPITB, observer A & VaSera VS-1500, observer A \\
VaSera VS-1500, observer B & VOPITB, observer B \\
VOPITB, observer A & VaSera VS-1500, observer A \\
VaSera VS-1500, observer B & VOPITB, observer B \\
\hline
\end{tabular}

Adapted from Wilkinson et al. ${ }^{20}$

value in metres $\left(\mathrm{kg} / \mathrm{m}^{2}\right)$. The waist circumference will be measured using a flexible graduated measuring tape with the patient in the standing position without clothing. BP will involve three measurements of systolic BP (SBP) and diastolic BP (DBP), and its value will be calculated as the mean values of the last two performed using a validated OMRON model HEM-907 sphygmomanometer (Omron Healthcare, Kyoto, Japan) according to the recommendations of the European Society of Hypertension. ${ }^{23} \mathrm{ABI}$ will be performed oscillometrically using VOPITB and VASERA.

\section{CAVI measurement}

The physical examination of the volunteers will be performed early in the morning. Subjects will be in the fasting condition and will be asked not to smoke for 1 hour before examination. They will rest in the lying position for at least $10 \mathrm{~min}$ before the measurement. All examinations will be conducted at least 12 hours after taking any vasoactive drugs. The values of CAVI will be determined from the measurements by VOPITB and VaSera VS-1500 (FukudaDenshi Co.). Six sequential recordings will be made by two operators, alternating the devices between recordings (three with VOPITB and three with VaSera VS-1500). The order of the device used in the measurements will also be alternated between subjects (table 1). The measurements will be taken 2-3 min apart to avoid reactive hyperaemia.

CAVI integrates information about the elasticity of blood vessels and represents a novel parameter of vascular stiffness that does not depend on BP. ${ }^{14}$ The mathematical expression to calculate CAVI values is described elsewhere $^{914}$ and is mainly based on substituting the stiffness parameters $\beta$ and PWV in the following equation: $\beta=2 \rho \times 1 /(\mathrm{SBP}-\mathrm{DBP}) \times \ln (\mathrm{SBP} / \mathrm{DBP}) \times \mathrm{PWV}^{2}$, where $\rho$ is the blood density and PWV is the heart-ankle PWV (haPWV) measured between the origin of the aorta and the tibial artery at the ankle. The mean coefficient of variation of CAVI is $<5 \%$, which is small enough for clinical use. Previous studies confirmed that CAVI has favourable intraobserver reproducibility at different times and interobserver reproducibility with different operators. ${ }^{9}$ The CAVI values, according to age and gender, are classified as normal $(\mathrm{CAVI}<8)$, borderline $(8 \leq \mathrm{CAVI}<9)$ and abnormal $(\mathrm{CAVI} \geq 9)$.
PWV measurement to calculate CAVI will be performed by VOPITB according to a previously described technique. ${ }^{17}$ Briefly, four cuffs are positioned on the two arms, near the elbow flexion crease, and on the two legs, close to the ankles. This device performs all functions automatically, including the cuffs inflating and recording pulse waves with an incorporated oscillometric sensor. The haPWV $(\mathrm{m} / \mathrm{s})$ is estimated using the following equation: heart-ankle length (Lha)/time. Time is the pulse transit time of the pulse wave from the origin of the aorta (peak of the $\mathrm{R}$ wave in lead II of an electrocardiographic record incorporated in the system) to its arrival at each of the extremities (the cuff placement). The Lha will be calculated by applying the next mathematical height-based formula: $\mathrm{Lhl}=0.8129 \times$ height $(\mathrm{cm})+12.328$. This measurement method was validated by VOPITB in the Spanish population $^{24}$ and is also validated by VaSera VS- 1500 . Because VOPITB can measure the haPWV in each limb, CAVI can be determined. The equation to calculate CAVI using the VOPITB device is described above $\left(\mathrm{CAVI}=2 \rho \times 1 /(\mathrm{SBP}-\mathrm{DBP}) \times \ln (\mathrm{SBP} / \mathrm{DBP}) \times \mathrm{haPWV}^{2}\right)$. The SI unit of BP will be used to convert haPWV to CAVI by VOPITB. Other CAVI measurements will be performed using VaSera VS-1500 according to the manufacturer's instructions. ${ }^{9}$ Cuffs will be fitted to the size of the arms and ankles of the patients. Electrodes will be attached to the two arms and two ankles, and a microphone for cardio-phonogram measurements will be fixed with double-sided tape over the sternum in the second intercostal space. CAVI measurements will be considered valid only when obtained during at least three consecutive heartbeats. ${ }^{14}$

\section{Statistical analysis}

For each subject, the average value of the three readings taken with each device will be calculated. The BlandAltman plot will be used to determine whether any bias exists in the data-that is, a tendency of the size of the difference to vary with the mean. ${ }^{20}$ The plot will show mean and \pm 2 SD. Ninety-five percent of the readings will lie between $\pm 2 \mathrm{SD}$ of the mean.

Continuous variables will be expressed as mean $\pm \mathrm{SD}$ for the case of normal distribution or as medians (IQR) for asymmetric distribution, and the categorical variables will be expressed as frequencies and percentages. The Kolmogorov-Smirnov test will be used to determine whether the continuous variables follow a normal distribution. The relationship of quantitative variables to each other will be tested using Pearson's or Spearman's correlation, as appropriate. Analysis of the difference of means between the continuous variables of two categories will be carried out using Student's t-test or the Mann-Whitney U test, as appropriate; categorical variables will be analysed using $\chi^{2}$ test. To evaluate the relationship between the continuous variables of more than two categories, analysis of variance and least significant difference test will be used in post hoc tests. The Kruskal-Wallis test will be used in cases where the variables are not normally distributed. 
A value of $\mathrm{p}<0.05$ will be considered statistically significant. The analyses will be performed using SPSS V.24.0 for Windows (SPSS).

\section{ETHICS AND DISSEMINATION}

The study designs were approved by the ethics committee of the Hospital San Pedro de Alcántara, Cáceres, Spain. The participants will be required to sign an informed consent form before inclusion in the study, in accordance with the Declaration of Helsinki and WHO standards for observational studies. ${ }^{22}$ The confidentiality of the subjects will be guaranteed at all times in accordance with the provisions of current legislation on personal data protection (15/1999 of 13 December Protection of Personal Data Official Law), and the conditions will be contemplated by Act 14/2007 on biomedical research.

The dissemination plan of the research study results will be through presentations in relevant national and international conferences and scientific publications in peer-reviewed journals.

\section{DISCUSSION}

CAVI is an alternative method to assess AS and overcomes some disadvantages of the gold standard method, that is, cfPWV. For example, it is easier to use, overcomes the problem of the acquisition of good carotid and femoral artery pulses, which is usually operator-dependent, and is sometimes challenging for clinical staff. ${ }^{25}$ In addition, CAVI is more comfortable for patients because the inguinal area is not exposed during the examination ${ }^{26}$ and the time needed to generate CAVI is usually less than that for the cfPWV measurements. Furthermore, cfPWV does not include the ascending aortic segment, which confirms the earliest changes during arterial ageing and disease. ${ }^{16}$ By contrast, CAVI reflects the stiffness from the ascending aorta to the ankle arteries and may be a comprehensive marker of systemic AS. ${ }^{27}$ Other potential advantages include that CAVI has low operator dependency and is less affected by BP at the time of measurement than $\mathrm{cfPWV} .{ }^{14}$ Moreover, CAVI may have higher reproducibility than PWV in several vascular beds. ${ }^{9}$ Currently, VaSera VS-1500 is the only commercial device that calculates CAVI. It is widely used in Asian countries and scarcely used in Europe (probably due to its high cost).

VOPITB calculates the PWV in the extremities using the oscillometric method, allowing the analysis of new PWV indices. ${ }^{17}$ It is simple to use and can correctly stratify patients according to their vascular risk. ${ }^{17-19}$ The AS variables provided by VOPITB make it a promising device for routine clinical practice in vascular risk evaluation in a large population. Incorporating CAVI into its performance (an AS variable of contrasted prognostic value) would complement the information provided by VOPITB and would make it a competitive device in the field of AS assessment. VOPITB is a European device, and its price will be lower than that of VaSera VS-1500. In addition, VOPITB determines more AS parameters than VaSera; thus, it will provide a more thorough evaluation of patients.

\section{Study limitations}

The inclusion of AS of leg arteries may be considered a caution of CAVI because elastic artery stiffness, but not muscular artery stiffness, seems to be independently associated with cardiovascular risk. ${ }^{4}$ Therefore, the inclusion of muscular arteries may contribute little to the prognostic value of CAVI. However, the practical implications of this issue in CVD risk assessments remain unclear and require future studies. In addition, baPWV (a parameter of AS including leg arteries) predicts CVD risk independently of traditional risk factors. ${ }^{28}$ Finally, the measurements used in this validation study will not be against an invasive method; however, PWV measured by VOPITB has already been validated with invasive techniques. ${ }^{17}$

\section{Author affiliations}

${ }^{1}$ Department of Nursing, Nursing and Occupational Therapy College. Universidad de Extremadura, Caceres, Spain

${ }^{2}$ Iberian Network on Arterial Structure, Central Hemodynamics and Neurocognition, Caceres, Spain

${ }^{3}$ Laboratory of Instrumentation, Biomedical Engineering and Radiation Physics (LIBPhys-UNL), NOVA School of Science and Technology, NOVA University Lisbon, Caprica, Portugal

${ }^{4}$ Zona Centro Health Center, Extremadura Health Service, Cáceres, Spain

${ }^{5}$ Deparment of Nursing, Faculty of Medicine, Universidad de Extremadura, Badajoz, Spain

${ }^{6}$ Department of Internal Medicine, Hospital San Pedro de Alcantara, Caceres, Spain

Contributors SRM, JFCG and JFSM-T have participated in the conception of the idea for the study. SRM, VV, JMdNJ, PRS, AS and MMA have participated in the design of the study, development of the protocol and writing of the manuscript. JFCG and JFSM-T have reviewed the manuscript.

Funding This study was funded by Consejería de Economía e Infraestructura de la Junta de Extremadura (Spain) (Grant no: IB16218).

Competing interests JFSM-T has property rights over the patented VOPITB invention (Oficina Española de Patentes y Marcas; number of concession: P201130872; publication number: 2400134) and is consultant for MSD, Astra, Pfizer and Boehringer Ingelheim.

Patient consent for publication Not required.

Provenance and peer review Not commissioned; externally peer reviewed.

Open access This is an open access article distributed in accordance with the Creative Commons Attribution Non Commercial (CC BY-NC 4.0) license, which permits others to distribute, remix, adapt, build upon this work non-commercially, and license their derivative works on different terms, provided the original work is properly cited, appropriate credit is given, any changes made indicated, and the use is non-commercial. See: http://creativecommons.org/licenses/by-nc/4.0/.

ORCID iD

Sergio Rico Martín http://orcid.org/0000-0003-4932-1940

\section{REFERENCES}

1 Vlachopoulos C, Aznaouridis K, Stefanadis C. Prediction of cardiovascular events and all-cause mortality with arterial stiffness: a systematic review and meta-analysis. J Am Coll Cardiol 2010;55:1318-27.

2 Cooper LL, Palmisano JN, Benjamin EJ, et al. Microvascular function contributes to the relation between aortic stiffness and cardiovascular events: the Framingham heart study. Circ Cardiovasc Imaging 2016;9. 
3 Kaess BM, Rong J, Larson MG, et al. Aortic stiffness, blood pressure progression, and incident hypertension. JAMA 2012;308:875-81.

4 Laurent S, Cockcroft J, Van Bortel L, et al. Expert consensus document on arterial stiffness: methodological issues and clinical applications. Eur Heart J 2006;27:2588-605.

5 Van Bortel LM, Laurent S, Boutouyrie P, et al. Expert consensus document on the measurement of aortic stiffness in daily practice using carotid-femoral pulse wave velocity. $J$ Hypertens 2012;30:445-8.

6 Frimodt-Møller M, Nielsen AH, Kamper A-L, et al. Reproducibility of pulse-wave analysis and pulse-wave velocity determination in chronic kidney disease. Nephrol Dial Transplant 2008;23:594-600.

7 Di lorio BR, Cucciniello E, Alinei P, et al. Reproducibility of regional pulse-wave velocity in uremic subjects. Hemodial Int 2010;14:441-6.

8 Sigrist MK, Chiarelli G, Levin A, et al. Pulse wave velocity measurements are reproducible in multiple trained observers: a short report. Nephron Clin Pract 2010;116:c60-4.

9 Shirai K, Utino J, Otsuka K, et al. A novel blood pressureindependent arterial wall stiffness parameter; cardio-ankle vascular index (CAVI). J Atheroscler Thromb 2006;13:101-7.

10 Yamashina A, Tomiyama H, Takeda K, et al. Validity, reproducibility, and clinical significance of noninvasive brachial-ankle pulse wave velocity measurement. Hypertens Res 2002;25:359-64.

11 Kawasaki T, Sasayama S, Yagi S, et al. Non-Invasive assessment of the age related changes in stiffness of major branches of the human arteries. Cardiovasc Res 1987;21:678-87.

12 Bramwell JC, Hill A V. The velocity of the pulse wave in man. Proc $R$ Soc L B 1922.

13 Schillaci G, Battista F, D'Abbondanza M, et al. The impact of the cardio-ankle vascular index on left ventricular structure and function. Eur Hear J 2017;19:B30-4.

14 Shirai K, Hiruta N, Song M, et al. Cardio-ankle vascular index (CAVI) as a novel indicator of arterial stiffness: theory, evidence and perspectives. J Atheroscler Thromb 2011;18:924-38.

15 Kubozono T, Miyata M, Ueyama K, et al. Clinical significance and reproducibility of new arterial distensibility index. Circ $J$ 2007;71:89-94.

16 Matsushita K, Ding N, Kim ED, et al. Cardio-ankle vascular index and cardiovascular disease: systematic review and meta-analysis of prospective and cross-sectional studies. J Clin Hypertens 2019;21:16-24.
17 Muñoz-Tsorrero JFS, Tardio-Fernandez M, Valverde-Valverde $\mathrm{JM}$, et al. Pulse wave velocity in four extremities for assessing cardiovascular risk using a new device. J Clin Hypertens 2014:16:378-84.

18 Rico Martín S, de Nicolás Jiménez JM, Moyano Calvente SL, et al. [Pulse wave velocity of the leg minus that of the arm measured with a custom device correlates to the coronary calcium quantification]. Rev Clin Esp 2016;216:191-7.

19 Sánchez Babaicoa C, Rico Martín S, Morales E, et al. JSM-T. [Pulse wave velocity brachial-ankle with a new device]. Rev Clin Esp 2020.

20 Wilkinson IB, McEniery CM, Schillaci G, et al. Artery Society guidelines for validation of non-invasive haemodynamic measurement devices: Part 1, arterial pulse wave velocity. Artery Res 2010.

21 Gómez-Sánchez M, Patino-Alonso MC, Gómez-Sánchez L, et al. Reference values of arterial stiffness parameters and their association with cardiovascular risk factors in the Spanish population. The EVA study. Rev Esp Cardiol 2020;73:43-52.

22 World Medical Association Declaration of Helsinki. Ethical principles for medical research involving human subjects. JAMA 2013;310:2191-4.

23 Williams B, Mancia G, Spiering W, et al. ESC/ESH guidelines for the management of arterial hypertension: the task force for the management of arterial hypertension of the European Society of cardiology and the European Society of hypertension: the task force for the management of arterial H. J Hypertens 2018;2018:1953-2041.

24 Sánchez Muñoz-Torrero JF, Calderón-García JF, De Nicolás-Jiménez $\mathrm{JM}$, et al. Automatic or manual arterial path for the Ankle-brachial differences pulse wave velocity. PLoS One 2018;13:e0206434.

25 Butlin M, Qasem A. Large artery stiffness assessment using SphygmoCor technology. Pulse 2017;4:180-92.

26 Tanaka H, Munakata M, Kawano Y, et al. Comparison between carotid-femoral and brachial-ankle pulse wave velocity as measures of arterial stiffness. J Hypertens 2009;27:2022-7.

27 Lim J, Pearman M, Park W, et al. Interrelationships among various measures of central artery stiffness. Am J Hypertens 2016;29:1024-8.

28 Ohkuma T, Ninomiya T, Tomiyama H, et al. Brachial-Ankle pulse wave velocity and the risk prediction of cardiovascular disease: an individual participant data meta-analysis. Hypertension 2017;69:1045-52. 